\title{
LR-90 prevents dyslipidaemia and diabetic nephropathy in the Zucker diabetic fatty rat
}

\author{
J. L. Figarola $\cdot$ S. Loera $\cdot$ Y. Weng $\cdot$ N. Shanmugam • \\ R. Natarajan $\cdot$ S. Rahbar
}

Received: 1 June 2007 / Accepted: 8 January 2008 / Published online: 4 March 2008

(C) Springer-Verlag 2008

\begin{abstract}
Aims/hypothesis Previous studies have shown that LR-90, a new inhibitor of AGE formation, prevented the development of experimental type 1 diabetic nephropathy. In this study, we examined the effects of LR-90 in the Zucker diabetic fatty (ZDF) rat, a model of type 2 diabetes and metabolic syndrome, and investigated the mechanisms by which it may protect against renal injury.

Methods Male ZDF rats were treated without or with LR-90 from age 13 to 40 weeks. Metabolic and kidney functions and renal histology were evaluated. AGE accumulation and the production of the receptor for AGE (AGER) were measured. Profibrotic growth factors, extracellular matrix proteins and intracellular signalling pathways associated with glomerular and tubular damage were also analysed.

Results LR-90 dramatically reduced plasma lipids in ZDF rats, with only modest effects on hyperglycaemia. Renal AGE, AGER and lipid peroxidation were all attenuated by LR-90. LR-90 significantly retarded the increase in albuminuria and proteinuria. This was associated with reduction in glomerulosclerosis and tubulointerstitial fibrosis, concomitant with
\end{abstract}

Electronic supplementary material The online version of this article (doi:10.1007/s00125-008-0935-x) contains supplementary material, which is available to authorised users.

J. L. Figarola $\cdot$ Y. Weng $\cdot$ N. Shanmugam $\cdot$ R. Natarajan $\cdot$

S. Rahbar $(\bowtie)$

Department of Diabetes, Endocrinology and Metabolism,

Gonda Building, City of Hope National Medical Center,

1500 E. Duarte Road,

Duarte, CA 91010, USA

e-mail: srahbar@coh.org

S. Loera

Department of Anatomical Pathology,

City of Hope National Medical Center,

Duarte, CA, USA marked inhibition of renal overproduction of TGF- $\beta 1$, connective tissue growth factor, fibronectin and collagen IV. Additionally, LR-90 downregulated the activation of key mitogen-activated protein kinases (MAPKs) and nuclear factor kappa B (NF-KB) in the renal cortex.

Conclusions/interpretation These results support our earlier studies on the renoprotective effects of LR-90 on type 1 diabetic nephropathy and provide further evidence that LR-90, an AGE inhibitor with pleiotrophic effects, may also be beneficial for the prevention of type 2 diabetic nephropathy, where multiple risk factors, such as hyperglycaemia, dyslipidaemia, obesity, insulin resistance and hypertension, contribute to renal injury.

Keywords AGE inhibition · Diabetic nephropathy Dyslipidaemia · Extracellular matrix · Glomerulosclerosis . Glycation · Oxidative stress $\cdot$ Renal fibrosis $\cdot$ Renal protection - Type 2 diabetes

$\begin{array}{ll}\text { Abbreviations } \\ \text { AGER } & \text { receptor for AGE } \\ \text { ARB } & \text { angiotensin II receptor blocker } \\ \text { CML } & N^{\varepsilon} \text {-(carboxymethyl)lysine } \\ \text { CTGF } & \text { connective tissue growth factor } \\ \text { ECM } & \text { extracellular matrix } \\ \text { MAPK } & \text { mitogen-activated protein kinase } \\ \text { NF-KB } & \text { nuclear factor-kappa B } \\ \text { PAMS } & \text { periodic acid-Schiff-methenamine silver } \\ \text { ZDF } & \text { Zucker diabetic fatty } \\ \text { ZL } & \text { Zucker lean }\end{array}$

Introduction

Diabetic nephropathy is one of the most serious complications of diabetes and its incidence is increasing dramatically 
worldwide. It is now the leading cause of end-stage renal failure and the requirement for chronic dialysis or renal replacement therapy $[1,2]$. The characteristic features of diabetic nephropathy include persistent albuminuria, a progressive decline in renal function and, histopathologically, mesangial expansion followed by glomerulosclerosis and interstitial fibrosis [3, 4]. Although the pathological and clinical indices of diabetic nephropathy are well described, the precise molecular mechanisms underlying its pathogenesis are not completely understood. Over the last decade, evidence has accumulated, implicating AGE and AGE-protein crosslinking as a major factor in the progression of diabetic nephropathy $[5,6]$. Several studies have shown that the extent of AGE formation, particularly AGE adducts in glomerular and tubulointerstitial compartments, correlates with the severity of diabetic nephropathy [7, 8]. AGE may contribute to diabetic tissue injury by a number of different pathways, including receptor-independent alterations of the extracellular matrix (ECM) architecture and protein cross-linking, as well as by modulating expression of various inflammatory genes, cytokines and growth factors through cell surface receptors, including the receptor for AGE (AGER) $[9,10]$.

The Zucker diabetic fatty (ZDF) rat is considered to be an excellent animal model of obesity and type 2 diabetes, presenting a physiological and metabolic profile similar to that seen in humans. The ZDF rat is characterised by hyperglycaemia, hyperinsulinaemia, hyperlipidaemia, both moderate hypertension and obesity, and progressive renal injury $[11,12]$. These rats develop albuminuria at about 14 weeks, begin exhibiting glomerulosclerosis by 18 to 20 weeks and have increasing proteinuria resulting in chronic renal insufficiency by 22 weeks of age relative to their genetic lean controls [1315]. The nephropathy in this model has been described as focal segmental glomerulosclerosis associated with glomerulomegaly and mesangial expansion [14, 15]. Previous studies have shown that the development of diabetic nephropathy in obese ZDF rats can be ameliorated by treatment with peroxisome proliferator-activated receptor-gamma agonists $[16,17]$, ACE inhibitors and angiotensin II receptor blockers (ARBs) [18, 19], vasopeptidase inhibitors [19, 20], antivascular endothelial growth factor antibodies [21] and antioxidants [15]. These intervention studies strongly suggest that the pathogenesis of renal damage in type 2 diabetes involves a complex interrelationship of metabolic and/or haemodynamic factors.

Previously, our group reported new classes of compounds as inhibitors of AGE formation and protein cross-linking $[22,23]$. More recently, several of these compounds were found effective in preventing the development of diabetic nephropathy in streptozotocin-induced diabetic animals [24, 25]. In the present study, we evaluated whether LR-90, one of the more powerful AGE inhibitors with other biochemical effects (lipid-lowering, antioxidant, anti-inflammatory)
$[22,23,26]$, can prevent dyslipidaemia and the development of renal dysfunction in experimental models of type 2 diabetes. We also investigated the effects of LR-90 on the production of prosclerotic and profibrotic growth factors and ECM proteins and their intracellular signalling pathways in the kidney to explore the possible mechanisms responsible for the renoprotective effects of LR-90 on diabetic nephropathy in ZDF rats.

\section{Methods}

Materials Unless otherwise noted, all chemicals and reagents were obtained from Sigma (St. Louis, MO, USA). The Nephrat albumin kit was from Exocell (Philadelphia, PA, USA). Monoclonal antibodies against AGE and TGF- $\beta 1$ were purchased from CosmoBio (Tokyo, Japan) and R\&D Systems (Minneapolis, MN, USA), respectively. Polyclonal anti-AGER and connective tissue growth factor (CTGF) antibodies were from Novus Biologicals (Littleton, CO, USA), while anti-phospho-p38 mitogen-activated protein kinase (MAPK), p38 MAPK, p44/42 MAPK (ERK 1/2), and anti nuclear factor-kappa $\mathrm{B}(\mathrm{NF}-\mathrm{kB})$ antibodies were purchased from Cell Signaling Technology (Beverly, MA, USA). Anti-fibronectin, anti-collagen IV and anti-histone H2B polyclonal antibodies were obtained from Abcam (Cambridge, MA, USA), Cedarlane Laboratories (Burlington, ON, Canada) and Upstate Biotechnology (Lake Placid, NY, USA), respectively. All other immunohistochemical reagents were obtained from DakoCytomation (Carpinteria, CA, USA) and Vector Laboratories (Burlingame, CA, USA).

Experimental animals All animal studies were carried out in compliance with policies outlined in The Guide for the Care and Handling of Laboratory Animals (NIH Publication No. 85-23) that have been approved by the City of Hope Animal Care Committee. Male ZDF ( $f a / f a$ ) and male Zucker lean $(+/+; \mathrm{ZL})$ rats were obtained at the age of 5 weeks from Charles River Laboratories (Wilmington, MA, USA). Animals were maintained with free access to Purina 5008 rat chow (Purina Mills, St Louis, MO, USA) and tap water. All animals were individually housed in a controlled room with a $12 \mathrm{~h}$ dark-light cycle. At the age of 13 weeks, the animals were divided into the following groups: (1) ZL rats $(n=6)$; (2) ZDF rats without treatment $(n=6)$; and (3) ZDF rats treated with LR-90 at $50 \mathrm{mg} / \mathrm{l}$ in their drinking water (ZDF90, $n=6$ ). The dose of LR-90 was chosen after previous experiments with type 1 diabetic nephropathy [25]. Food, water intake, body weight and blood glucose levels were monitored periodically. Systolic blood pressure was measured by tail-cuff plethysmography (CODA2 Blood Pressure System; Kent Scientific, Torrington, CT, USA). Animals were killed at the age of 40 weeks by over- 
anaesthetisation with isoflurane and cardiac puncture. Blood samples were collected from each animal and transferred accordingly into heparinised vacutainer tubes; they were later centrifuged $\left(1,500 \times g\right.$ for $10 \mathrm{~min}$ at $\left.4^{\circ} \mathrm{C}\right)$ for plasma isolation. The kidneys were removed immediately, rinsed in PBS and weighed, after which specimens were snap-frozen in liquid nitrogen or fixed in $10 \%$ (vol./vol.) neutral buffered formalin for further biochemical and histological analyses.

Plasma glucose, lipids and $N^{\varepsilon}$-(carboxymethyl)lysine- $A G E$ Plasma glucose was measured using a glucose analyser (2300 STAT; YSI, Yellowsprings, OH, USA). Total plasma triacylglycerol and cholesterol concentrations were quantified using the Vitros 250 Chemistry System (Johnson \& Johnson, Rochester, NY, USA). $N^{\varepsilon}$-(Carboxymethyl)lysine (CML)-AGE was liberated from plasma proteins by acid hydrolysis and then measured by ion-pair reversed-phase liquid chromatography/tandem mass spectrometry as described previously [24].

Renal function Renal function was assessed by measuring urinary albumin and total protein excretion. Prior to killing, rats were housed in metabolism cages for $24 \mathrm{~h}$ urine collection. Urinary albumin was quantified using the Nephrat kit according to manufacturer's instructions. Total urinary protein was measured using a kit (DC Assay; Bio-Rad Laboratories, Hercules, CA, USA).

Kidney histopathology Kidney sections were stained with periodic acid-Schiff-methenamine silver (PAMS) to evaluate glomerulosclerosis and mesangial matrix expansion; Masson's trichrome staining was used to analyse collagen deposition and cortical tubule degeneration. Glomerulosclerosis was defined as glomerular basement membrane thickening, mesangial hypertrophy and capillary occlusion, and was semiquantitatively evaluated on an arbitrary scale of 0 to 4 : grade 0 (normal); grade 1 (sclerotic area less than $25 \%$ ); grade 2 (sclerotic area $25-50 \%$ ); grade 3 (sclerotic area 50-75\%); and grade 4 (sclerotic area more than $75 \%$ ). The glomerulosclerotic index was then calculated as described [27]. Briefly, 100 glomeruli were randomly chosen from each rat kidney stained with PAMS and carefully scored for sclerosis by two blinded investigators. This was done in a sequential manner to ensure that the same glomerulus was not graded twice. To quantify for mesangial expansion and ECM accumulation in the mesangial areas, PAMS-positive materials in the mesangial region excluding cellular elements were analysed using an image analyser with a microscope (Olympus AX70; Olympus Optical, Hamburg, Germany) and photographed using a Retiga 2000R CCD camera (Qimaging, Surrey, BC, Canada). Twenty glomeruli, randomly selected from each rat were evaluated by two investigators without knowledge of the origin of the slides. The percentage of PAMS-positive area in each glomerulus was analysed using Image Pro Plus (Media Cybernetics, Silver Spring, MA, USA). Results were expressed as percentage of areas positive for PAMS staining. To evaluate cortical tubule degeneration and collagen deposition, kidney sections were stained with Masson's trichrome. In each section, six fields of kidney cortex were randomly selected at 100× magnification using an Olympus AX70 microscope, photographed using a Retiga 2000R camera and the images analysed by Image Pro Plus. Blue and red colours indicate collagen and cytoplasm staining, respectively. Degenerate tubules in each field were identified by the absence of cytoplasm and results expressed as number of degenerate tubules per field [27]. For quantitative measurement of collagen deposition, positively stained areas from six random regions in each slide were identified and scored by dividing the positively stained areas by the total tissue area in the same field using the colour separation function of Image Pro Plus. Results were expressed as percentage of area with positive staining.

Immunohistochemistry Tissue sections were deparaffinised by sequential exposure to xylene and ethanol, dehydrated and then exposed to $\mathrm{H}_{2} \mathrm{O}_{2}$ to eliminate endogenous peroxidase activity. The kidney sections were then blocked with $2 \%$ (wt/vol.) BSA and exposed to specific antibodies against AGE, AGER, TGF- $\beta 1$, CTGF, fibronectin and collagen type IV. Biotinylated anti-rabbit or anti-mouse $\operatorname{IgG}$ was then applied as a secondary antibody, followed by horseradish peroxidaseconjugated streptavidin. The staining was visualised by reaction with 3,3'-diaminobenzidine tetrahydrochloride and then counterstained with haematoxylin. All the sections were examined by light microscopy (Olympus AX70) and photographed with Retiga 2000R camera.

Tissue extraction Renal cortical tissues were homogenised in PBS buffer with 1\% (vol./vol.) Nonidet P-40, 0.5\% (wt/vol.) sodium deoxycholate, $0.1 \%$ (wt/vol.) SDS and protease inhibitor mixture (Roche, Indianapolis, IN, USA). Homogenates were centrifuged for $15 \mathrm{~min}$ at $4^{\circ} \mathrm{C}$ and at $12,000 \mathrm{rpm}$ and the supernatant fractions collected and stored at $-70^{\circ} \mathrm{C}$. Nuclear extracts were prepared as described previously [28]. Protein concentrations were measured using the Bio-Rad DC Assay kit. Total RNA was isolated using the RNA-STAT-60 reagent (Tel-Test, Friendswood, TX, USA).

Western blot assay Protein samples $(50 \mu \mathrm{g})$ were separated on 10 to $20 \%$ gradient Tris-glycine Criterion gels (Bio-Rad) and then transferred to nitrocellulose membranes. The membranes were blocked with 5\% (wt/vol.) skimmed milk in PBS before incubation with anti-TGF- $\beta 1$, anti-CTGF, anti-AGER, anti-phospho-p38 MAPK, anti-phospho-p44/42 MAPK and anti-p65 NF-KB antibodies. Immunoreactive proteins were detected by enhanced chemiluminescence (Western Lightning 
Chemiluminescence Reagent; Perkin-Elmer, Waltham, MA, USA) using horseradish peroxidase-labelled secondary antibodies. Equal amounts of protein loading were confirmed by restaining the membranes with antibodies against $\beta$-actin, p38 MAPK or p44/42 MAPK for cytosolic extracts or with histone H2B for nuclear extracts. Band intensities were quantified using a densitometer (Quantity One, Bio-Rad).

Reverse transcriptase-polymerase chain reaction Total RNA $(1 \mu \mathrm{g})$ was used for the RT reaction using the GeneAmp RNA PCR kit (Applied Biosystems, Foster City, CA, USA). Total cDNA was then used in PCR reactions containing genespecific primers [Electronic supplementary material (ESM) Table 1] paired with Quantum 18S rRNA internal standards (Ambion, Austin, TX, USA). Multiplex PCR reactions were performed in a GeneAmp 9700 (Applied Biosystems). PCR products were fractionated on $2 \%$ (wt/vol.) agarose gel electrophoresis and photographed using Alphalmager 2000 (Alpha Innotech, San Leandro, CA, USA). The densities of amplified products corresponding to specific genes and $18 \mathrm{~S}$ rRNA were quantified with Quantity One software (Bio-Rad). Results are expressed as the means of the ratios of $T g f b 1$, Ctgf or Ager band densities to the 18S rRNA band density.

Kidney lipid peroxidation To assess lipid peroxidation in renal cortical homogenates, malondialdehyde, an end-product of lipid peroxidation, was measured as thiobarbituric acidreactive substances by colorimetric reaction as described [29]. Reaction was carried in the presence of EDTA and antioxidant butylated hydroxytoluene, and the absorbance was measured at $532 \mathrm{~nm}$ (UV-1601; Shimadzu Scientific Instruments, Columbia, MD, USA). Hydrolysed 1,1,3,3-tetramethoxypropane was used as standard [prepared by incubating $10 \mathrm{mmol} / \mathrm{l}$ tetramethoxypropane with $1 \%$ (vol./vol.) $\mathrm{H}_{2} \mathrm{SO}_{4}$ for $2 \mathrm{~h}$ at room temperature]. Results are expressed as nanomole malondialdehyde per milligram protein.

Data analyses Statistical analyses were performed using Prism (GraphPad, San Diego, CA, USA). Data were first

Table 1 Metabolic data on Zucker lean, diabetic fatty and LR-90treated ZDF rats at 40 weeks of age

\begin{tabular}{llll}
\hline & $\mathrm{ZL} \dagger$ & $\mathrm{ZDF} \dagger$ & $\mathrm{ZDF90} \dagger$ \\
\hline Body weight $(\mathrm{g})$ & $448 \pm 5$ & $425 \pm 18$ & $347 \pm 32$ \\
Plasma glucose $(\mathrm{mmol} / \mathrm{l})$ & $9.6 \pm 0.5^{* *}$ & $29.6 \pm 1.3$ & $25.0 \pm 2.3$ \\
Plasma triacylglycerol (mmol/1) & $1.9 \pm 0.2^{* *}$ & $9.8 \pm 2.1$ & $4.4 \pm 0.6^{*}$ \\
Plasma cholesterol (mmol/l) & $2.5 \pm 0.2^{* *}$ & $8.9 \pm 0.9$ & $6.3 \pm 0.3^{*}$ \\
Mean systolic BP (mmHg) & $140 \pm 5^{*}$ & $158 \pm 5$ & $144 \pm 6$ \\
Heart rate (beats per min) & $340 \pm 11$ & $341 \pm 13$ & $330 \pm 12$ \\
\hline
\end{tabular}

Values are means \pm SEM

${ }^{*} p<0.05, * * p<0.01$ vs ZDF rats

$\dagger n=6$

ZDF90, LR-90-treated ZDF rats analysed by ANOVA and post hoc comparisons between group means analysed using two-tailed unpaired $t$ test. The strength of the relationship between two variables was assessed by the Pearson correlation test. A $p$ value of less than 0.05 was considered statistically significant. Data are presented as means \pm SEM.

\section{Results}

Metabolic parameters Table 1 shows the metabolic data of ZL, ZDF and LR-90 treated ZDF (ZDF90) rats at age 40 weeks. As expected, ZDF rats were hyperglycaemic, hyperlipidaemic, polydipsic and had slightly elevated blood pressure in comparison with their lean ZL counterparts. Body weights and heart rates of the three treatment groups at the time of death were not significantly different from each other. Administration of LR-90 to ZDF rats significantly reduced plasma triacylglycerol and cholesterol by $\sim 55 \%$ and $\sim 30 \%$, respectively $(p<0.05)$. LR-90 had only modest, but not statistically significant effects on hyperglycaemia and blood pressure. In addition, body weight was lower in LR90 treated ZDF rats than in ZDF controls, although the difference was not statistically significant $(p=0.061)$.

Renal function and histology ZDF rats had increased urinary volume output, total urinary protein and albumin excretion compared with lean controls (Table 2). Also, ZDF rats had higher kidney weights (both mean and relative weights) than ZL rats $(p<0.001)$, indicating nephromegaly. In addition, kidneys of ZDF rats exhibited increased mesangial ECM accumulation, glomerulosclerosis and tubulointerstitial injury as evidenced by excessive accumulation of PAMS-positive materials in the mesangial area of the glomeruli (Fig. 1a,c), resulting in higher glomerulosclerotic index scores (Fig. 1d), increased interstitial and glomerular collagen deposition, and tubular degeneration in the renal cortex (Fig. 1b,e,f). Treatment

Table 2 Renal function data of Zucker lean, diabetic fatty and LR-90treated ZDF rats at 40 weeks of age

\begin{tabular}{|c|c|c|c|}
\hline & $\mathrm{ZL} \dagger$ & $\mathrm{ZDF} \dagger$ & ZDF90† \\
\hline Urine volume (ml/day) & $19 \pm 3 * *$ & $175 \pm 22$ & $137 \pm 24$ \\
\hline $\begin{array}{l}\text { Urinary albumin } \\
(\mathrm{mg} / 24 \mathrm{~h})\end{array}$ & $1.3 \pm 0.4 * *$ & $44.7 \pm 3.8$ & $25.8 \pm 4.8^{*}$ \\
\hline $\begin{array}{l}\text { Total urinary protein } \\
(\mathrm{mg} / 24 \mathrm{~h})\end{array}$ & $22.2 \pm 3.4^{* *}$ & $529.7 \pm 122.7$ & $176.4 \pm 43.3^{* *}$ \\
\hline Mean kidney weights (g) & $2.74 \pm 0.05^{* *}$ & $4.79 \pm 0.30$ & $4.23 \pm 0.27$ \\
\hline $\begin{array}{l}\text { Relative kidney weights } \\
(\mathrm{g} / \mathrm{kg})\end{array}$ & $6.13 \pm 0.14 * *$ & $11.26 \pm 0.43$ & $12.23 \pm 0.78$ \\
\hline
\end{tabular}

Values are means \pm SEM

${ }^{*} p<0.05, * * p<0.01$ vs ZDF rats

$\dagger n=6$

ZDF90, LR-90-treated ZDF rats 

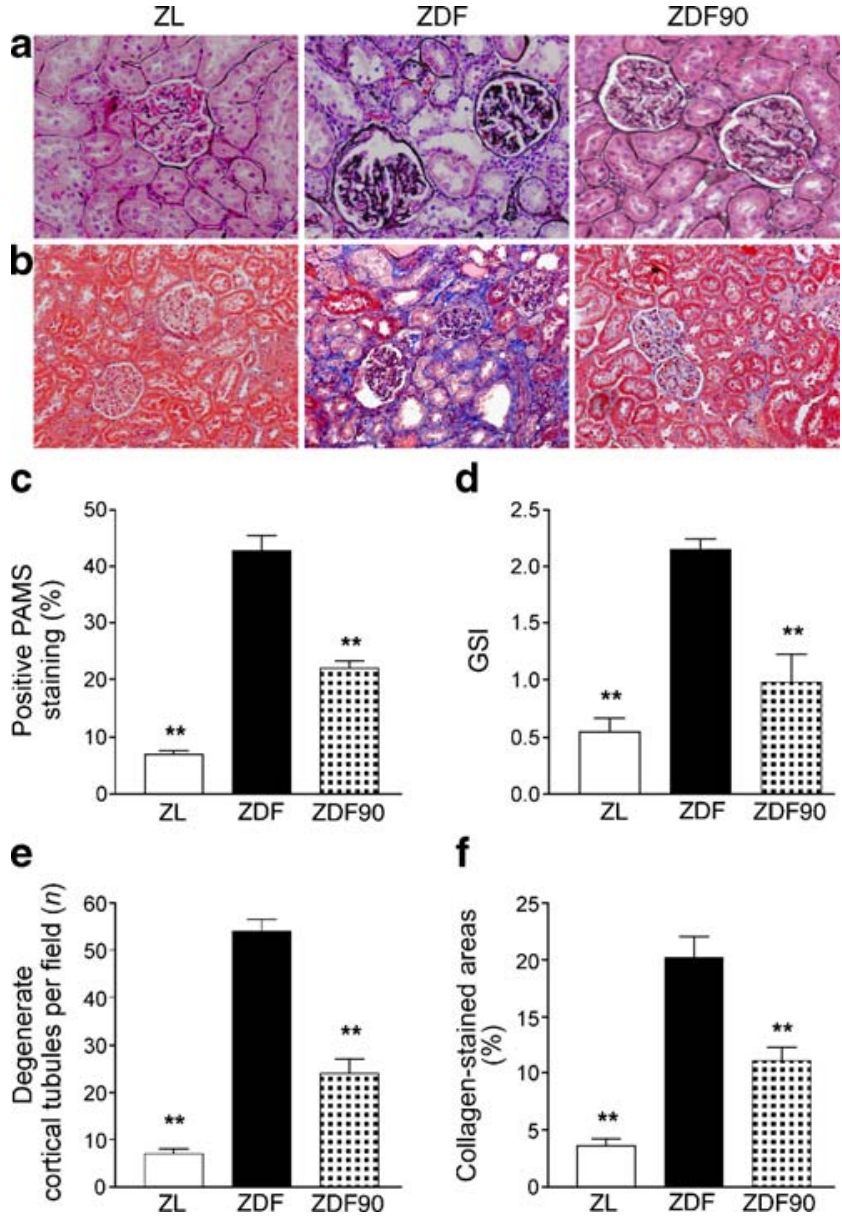

Fig. 1 LR-90 ameliorates renal injury in ZDF rats. Representative photomicrographs of (a) PAMS staining (original magnification, $\times 320$ ) showing increased positive staining inside glomeruli in ZDF rats and its reduction by LR-90, and (b) Masson's trichrome staining (original magnification, $\times 100$ ) demonstrating tubular degeneration and interstitial and glomerular collagen deposition in ZDF rats and its attenuation by LR-90. Quantitative scoring for (c) PAMS staining, (d) glomerulosclerosis index (GSI), (e) tubular degeneration and (f) percentage positive collagen area staining were all reduced significantly by LR-90 treatment. ${ }^{* *} p<0.01$ vs ZDF rats $(n=4-6)$. ZDF90, LR-90-treated ZDF rats

of ZDF rats with LR-90 significantly prevented an increase in urinary albumin $(42 \%, p<0.05)$ and total protein $(67 \%$, $p<0.01$ ), but not in urine output and kidney weights (Table 2). Reductions in both albumin and total protein excretion were strongly associated with attenuation of glomerulosclerosis and mesangial ECM accumulation (Fig. 1a,c,d), cortical tubule degeneration (Fig. 1b,e) and renal collagen deposition (Fig. 1b,f).

Renal TGF- $\beta 1, C T G F$ and ECM proteins Both TGF- $\beta 1$ and CTGF growth factors have been implicated in the pathogenesis of diabetic nephropathy, specifically in ECM accumulation and renal fibrosis. To examine whether LR-90 also affects the production of ECM proteins and fibrogenic cytokines, RT-PCR, Western blotting and immunostaining were performed. $T g f b 1$ and Ctgf mRNA (Fig. 2a,b) and protein (Fig. 2c-e) were significantly increased in kidneys of ZDF rats compared with ZL animals. Correspondingly, immunostaining for TGF- $\beta 1$ was increased in the glomerular, epithelial and mesangial cells as well as in the tubulointerstitium of ZDF rats (Fig. 3a). CTGF immunostaining was also increased in ZDF rats, this being more prominent in the tubulointerstitial areas (Fig. 3b). Treatment with LR-90 significantly attenuated both mRNA expression and protein production of TGF- $\beta 1$ and CTGF in the kidneys of ZDF animals (Figs 2a-e, 3a,b). Examination of fibronectin and collagen IV by immunohistochemical staining indicated that both ECM proteins could be lightly observed in the kidneys of ZL rats; fibronectin staining was mostly in the intraglomerular mesangium (Fig. 3c), with collagen IV weakly stained in the mesangium and tubulointerstitium (Fig. 3d). Both the intensity and area of fibronectin and collagen IV staining were dramatically increased in the ZDF rats compared with $Z \mathrm{~L}$ animals, this increase being strongly associated with increased PAMS-positive materials in the
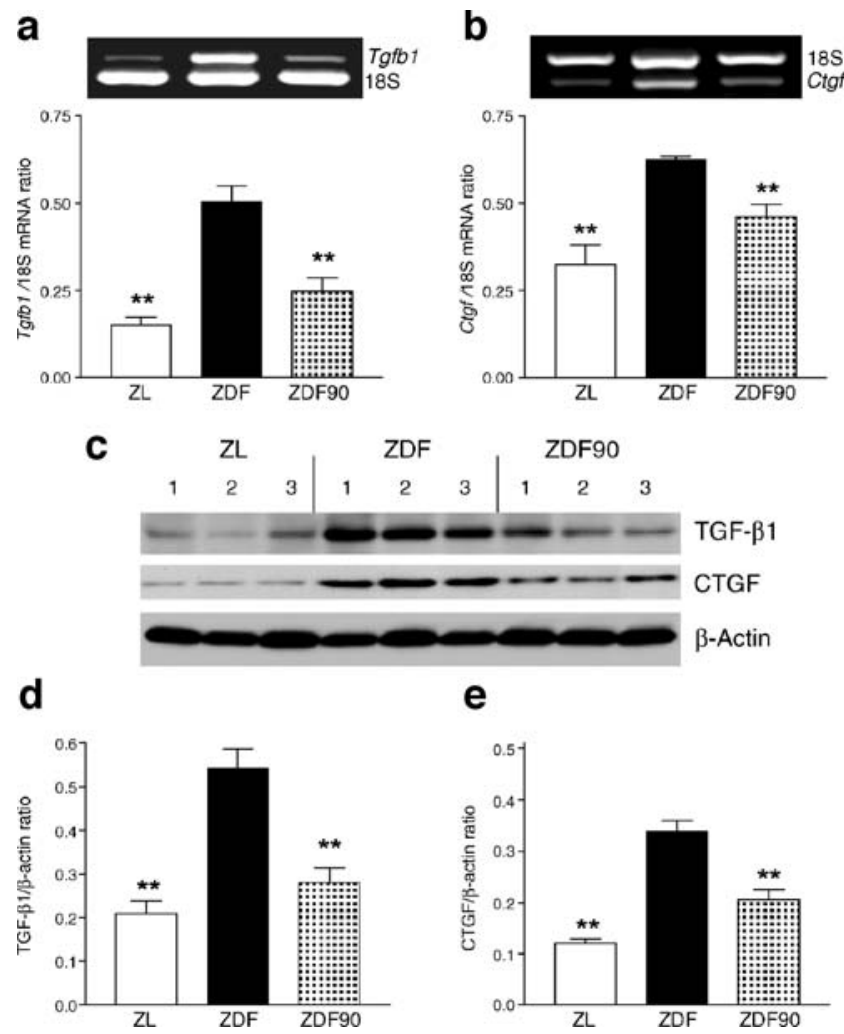

Fig. $2 T g f b 1$ and $C t g f$ mRNA expression and protein production were reduced by LR-90 treatment. Representative gel profiles (a, b) for RTPCR with quantitative densitometric analyses showing the effects of LR-90 on mRNA expression. c Representative western blot showing protein production in the renal cortex, with (d, e) quantitative densitometric analyses. Values in bar graphs: mean \pm SEM $(n=3-4)$. $* * p<0.01$ vs ZDF. ZDF90, LR-90-treated ZDF rats 


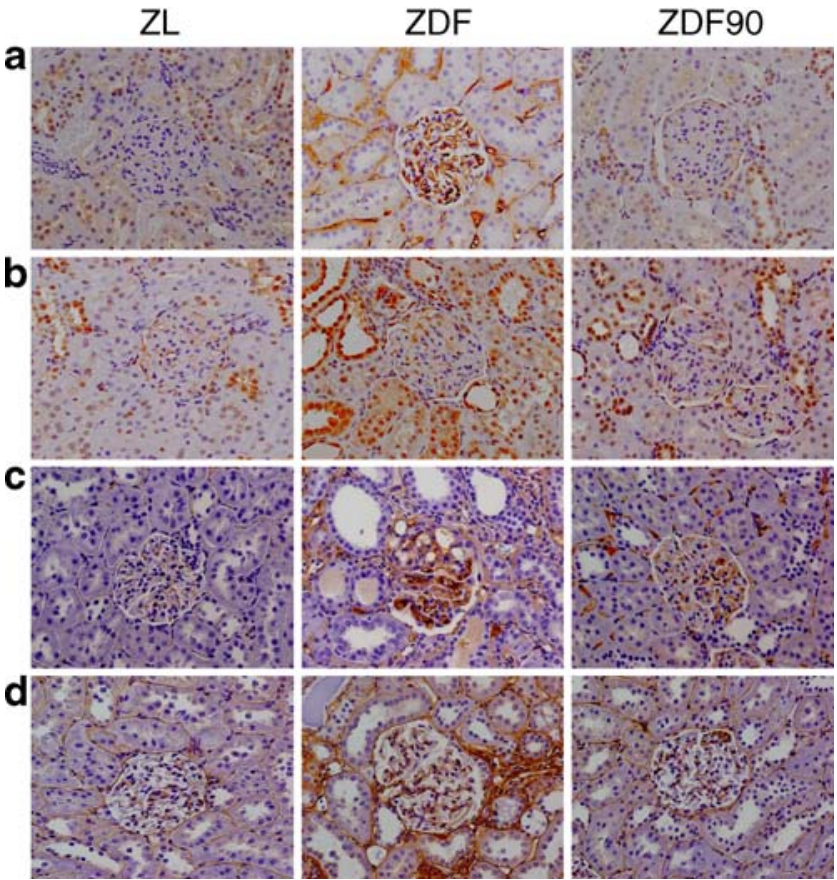

Fig. 3 Renal immunohistochemical stainings for TGF- $\beta 1$, CTGF and ECM proteins. Representative photomicrographs of immunostaining for (a) TGF- $\beta 1$, (b) CTGF, (c) fibronectin and (d) collagen IV, showing the marked reduction of positive area staining for these growth factors and ECM proteins in ZDF rats treated with LR-90. Original magnification, $\times 320$. ZDF90, LR-90-treated ZDF rats

glomeruli (Fig. 1a). Treatment with LR-90 markedly reduced the positive immunostaining for both ECM proteins to almost similar levels as those in ZL rats (Fig. 3c,d).

Renal MAPKs and $N F-\kappa B$ activation Pathways analyses have demonstrated that activation of MAPKs and NF-KB are key intracellular signals that play important roles in the progression of diabetic nephropathy, with the latter as important mediator of the inflammatory pathways [30]. We therefore analysed the activation of key growth and stressrelated MAPKs and the redox-sensitive transcription factor $\mathrm{NF}-\mathrm{KB}$ in the cortical tissues of experimental rats using western blotting. Compared with ZL animals, ZDF rats exhibited robust activation of $\mathrm{p} 38$ and p44/42 MAPKs, as assessed by an increase in the levels of phosphorylation of their corresponding phosphorylated proteins. Similarly, activation of p65 subunit of NF-kB was 2.3-fold higher in kidneys of ZDF rats than in those of ZL rats. Treatment with LR-90 significantly attenuated the activation of p38 $(p<0.05)$ and $\mathrm{p} 44 / 42(p<0.01)$ MAPKs, as well as NF-KB $(p<0.01$; Fig. 4a,b).

Plasma and renal $A G E$ and $A G E R$ Circulating plasma CML-AGE concentration was increased 1.5-fold in ZDF rats, which was significantly attenuated by LR-90 treatment $(22 \%$, $p<0.01$; Fig. 5a). A significant correlation between plasma CML-AGE and albuminuria was observed $(r=0.82, p<$ 0.0001; Fig. 5b). Subsequent immunohistochemical analysis of CML-AGE formation in the renal cortex showed a marked increase in AGE staining in the glomeruli and the cortical tubules, as well as in interstitium (Fig. 5c). AGER immunostaining, primarily in the cortical tubules and interstitium, was also increased in the ZDF rats (Fig. 5d). This enhanced AGER staining was strongly associated with increased Ager expression (Fig. 5e). LR-90 treatment markedly abrogated both AGE accumulation and Ager mRNA and protein in kidney tissues of ZDF rats (Fig. 5a,c-e).

Kidney lipid peroxidation Renal tissue malondialdehyde concentrations were increased 2.5-fold in ZDF rats compared with lean rats (Fig. 6a), which correlated strongly with albumin excretion rates $(r=0.81, p<0.0001$; Fig. $6 \mathrm{~b})$. Treatment with LR-90 substantially reduced kidney lipid peroxidation in ZDF animals by almost $40 \%$ (Fig. 6a).
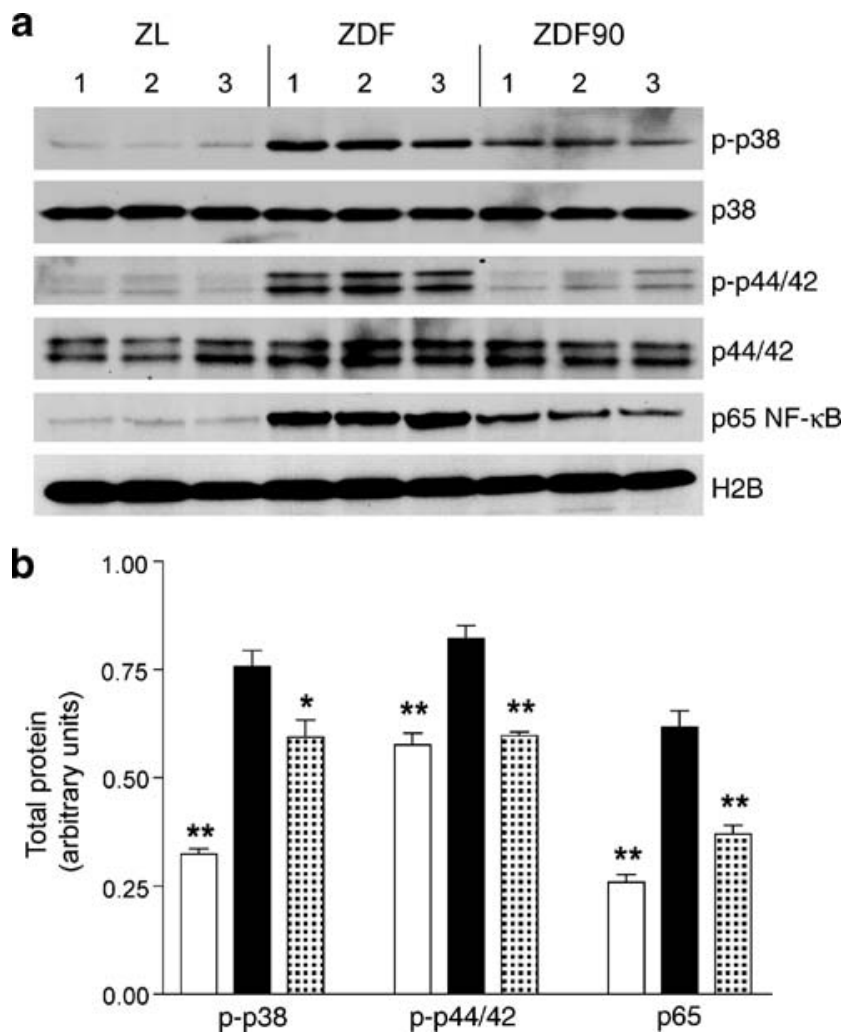

Fig. 4 LR-90 significantly attenuated renal MAPKs and NF-kB activation. Western blots (a) showing that the increased phosphorylation of p38 MAPK and p44/42 MAPK in the cortical lysates and activation of $\mathrm{p} 65$ subunit of NF- $\mathrm{KB}$ in the nucleus were significantly reduced by LR-90 treatment. Graph (b) shows the densitometric analyses (mean \pm SEM) of the ratio of p-p38 and p-p44/42 MAPK to total p38 MAPK and p44/42 MAPK, respectively and p65 to histone H2B. White bars, ZL; black bars, ZDF; chequered bars, ZDF90. ${ }^{*} p<0.05$, ${ }^{* *} p<0.01$ vs ZDF ( $n=3)$. p, phospho; ZDF90, LR-90-treated ZDF rats 

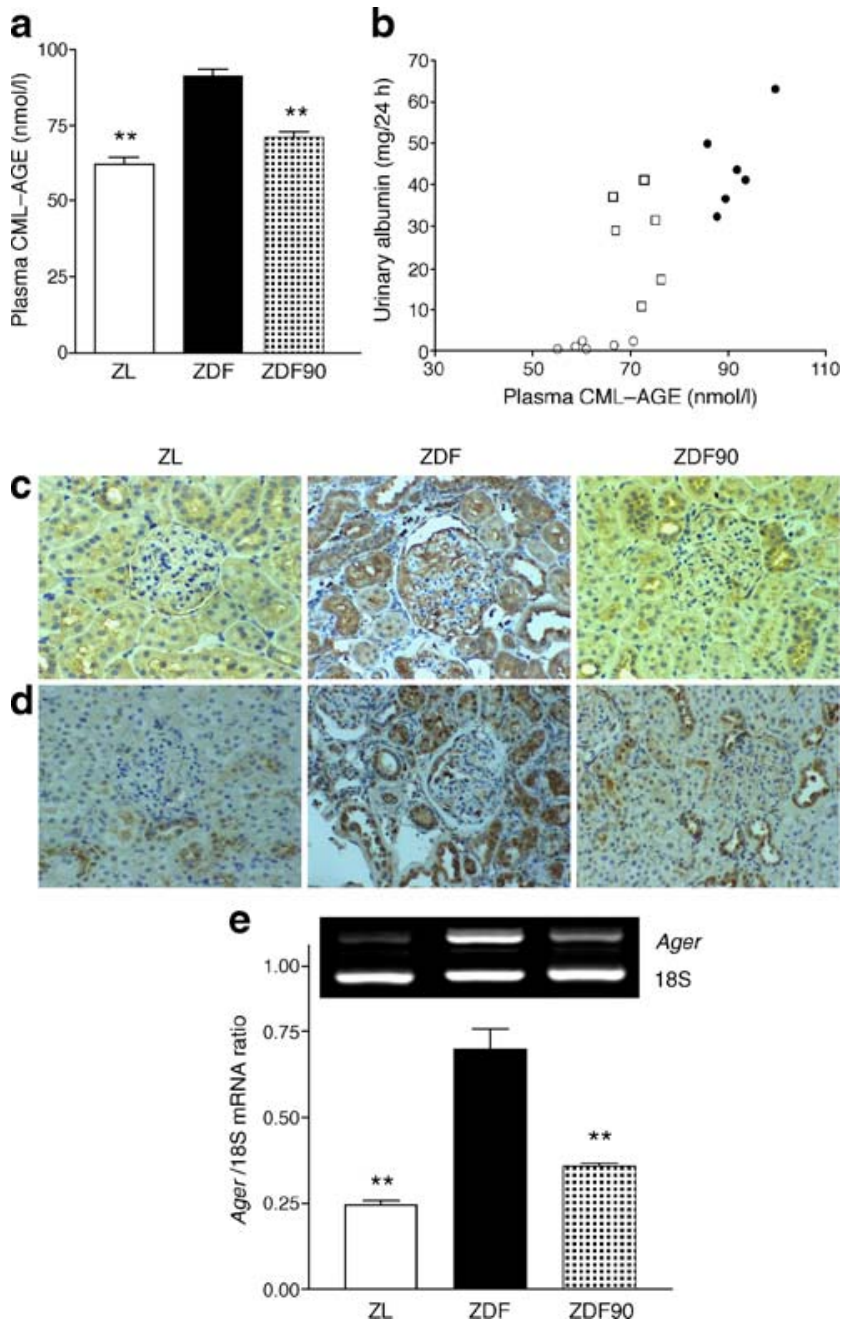

Fig. 5 LR-90 inhibits AGE formation and AGER activation. Circulating plasma CML-AGE (a) was measured using ion-pair reversed-phase liquid chromatography/tandem mass spectrometry. Correlation analysis (b) showed strong association between CMLAGE and urinary albumin excretion $(r=0.82, p<0.0001$, if data were merged from all three groups). White circles, ZL; black circles, ZDF; white squares, ZDF90. Immunohistochemical stainings demonstrated that renal AGE (c) and (d) AGER protein formation in ZDF rats were also reduced by LR-90 treatment. Original magnification $\times 320$. Expression of renal Ager, as determined by RT-PCR (e), was also suppressed by LR-90. ${ }^{* *} p<0.01$ vs ZDF $(n=4)$. ZDF90, LR-90treated ZDF rats

\section{Discussion}

Preventing or delaying the progression of diabetic nephropathy has been a major goal in biomedical research. Current therapeutic approaches such as ACE inhibitors and ARBs are clearly of value in reducing progression of established diabetic kidney disease [31]. However, the epidemic rise in the number of patients with end-stage renal disease due to diabetes continues, even though ACE inhibitors and ARBs are widely prescribed [32]. To develop innovative thera- peutic alternatives for combating diabetic nephropathy, a comprehensive understanding of the pathophysiology of this disease is imperative. In addition, targeting multiple risk factors associated with the prevention and progression of type 2 diabetic nephropathy seems more likely to be beneficial, as shown by recent approaches [33, 34].

Our current studies demonstrate the possibility of preventing diabetic nephropathy in a rat model of type 2 diabetes using LR-90, a compound that was initially identified as an AGE inhibitor and has recently been shown to exhibit other properties such as metal chelator, antioxidant, free radical scavenger and anti-inflammatory compound [22-24]. Consistent with earlier studies on streptozotocin-diabetic rats [26], LR-90 significantly reduced albuminuria and prevented kidney damage in the ZDF rats by limiting glomerular and tubular injuries. These benefits occurred in the context of reduced renal AGE accumulation and AGER production, with modest, but not statistically significant effects on hyperglycaemia and the moderate increase in blood pressure characteristic of ZDF rats. Furthermore, LR-90 provided additional beneficial effects by lowering plasma lipids and preventing lipid peroxidation in renal tissues. These findings suggest that even in type 2 diabetes, where obesity and hyperglycaemia combined with dyslipidaemia, increased insulin resistance and hypertension result in enhanced actions of several risk factors for organ damage, LR-90 was able to attenuate renal injury by modulating several metabolic factors involved in the pathogenesis of diabetic nephropathy. More recently, we also observed similar renoprotective effects of LR-90 on $d b / d b$ mice (S. Rahbar and J. L. Figarola, unpublished observations), further supporting the bioefficacy of this compound in preventing the development of type 2 diabetic nephropathy.
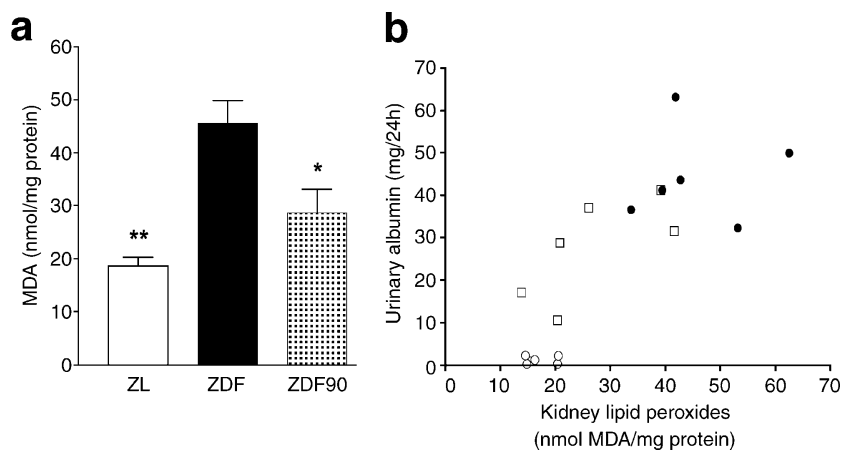

Fig. 6 LR-90 prevents renal lipid peroxidation. Kidney lipid peroxidation (a) was quantified by measuring malondialdehyde (MDA) in tissue homogenates using the thiobarbituric acid-reactive substances method. ${ }^{*} p<0.05, *^{*} p<0.01$ vs ZDF. b Correlation analysis showing strong association between kidney lipid peroxidation and urinary albumin excretion $(r=0.81, p<0.0001$ if data were merged from all three groups). White circles, ZL; black circles, ZDF; white squares, ZDF90. ZDF90, LR-90-treated ZDF rats 
It has been suggested that diabetic nephropathy occurs as a result of interactions between metabolic and haemodynamic factors, which activate common pathways leading to increasing proteinuria, glomerulosclerosis and tubulointerstitial fibrosis [35]. The haemodynamic factors act independently or in concert with metabolic factors to activate intracellular second messengers such as protein kinase $\mathrm{C}$ and MAPKs, NF- $\mathrm{BB}$ and various growth factors, which work in a coordinated manner to promote increased production and deposition of ECM proteins such as collagen, fibronectin and laminin. ECM accumulation in the glomeruli and tubulointerstitial fibrosis have been considered to be principal hallmarks of diabetic nephropathy and result from an imbalance between signalling activities of the different growth factors involved in renal matrix homeostasis [36]. TGF $\beta$-dependent and independent upregulation of CTGF and associated downstream signalling pathways appear to be important prosclerotic and profibrotic events in ECM regulation, as both TGF- $\beta 1$ and CTGF have been demonstrated to contribute to the pathology of diabetic nephropathy in studies with experimental and human diabetes, as well as with glomerular cells $[37,38]$. Production of these growth factors has been previously shown to be regulated by AGE-AGER activation [39, 40]. In our present study, ECM accumulation and renal fibrosis, along with increased $T g f b 1$ and $C \operatorname{tg} f$ mRNA expression and protein production, were observed in ZDF rats, results that were significantly attenuated by LR-90 treatment. Moreover, glomerular and tubular injuries were also minimised by the compound, with concomitant improvement in renal function in ZDF rats. Taken together, these results indicate that aside from inhibiting circulating and tissue AGE accumulation and AGER activation in the diabetic kidney, LR-90 treatment could also influence various intracellular signalling pathways associated with mesangial ECM accumulation and tubulointerstitial fibrosis.

Pathways analyses have demonstrated that MAPK activation, including p38 and p44/42 MAPK signalling pathways, are profibrotic contributors to diabetic nephropathy [41, 42]. These intracellular signals have also been shown to be partly mediated by AGE-AGER interaction and subsequent oxidant stress [43, 44]. Thus, in further elucidating possible mechanisms of the pharmacological effects of LR-90 against renal fibrosis in ZDF rats, we also demonstrated that LR-90 downregulated the activation of MAPKs p38 and p44/42 in renal cortex. Furthermore, we also showed that LR-90 inhibited the activation of the redox-sensitive transcription factor NF- $\mathrm{B}$, suggesting that LR-90 may also mediate the inflammatory reactions and associated gene expression of various cytokines involved in the pathogenesis of diabetic nephropathy. Our recent studies in monocytes further confirm this action of LR-90, where suppression of NF- $\mathrm{KB}$ activation by the compound was associated with its antioxidant activities against NADPH-mediated superoxide generation [26]. Indeed, accumulating evidence in vitro suggests that the response of mesangial cells, as well as tubular epithelial cells to hyperglycaemia is driven primarily by reactive oxygen species [36, 45]. More recent studies on ZDF rats seem to support this concept, arguing that oxidative stress is precedent to renal injury in this animal model $[15,46]$. Although we only performed limited tests to evaluate the oxidant status of ZDF rats treated with LR-90 (i.e. kidney lipid peroxidation), it is logical, on the basis of: (1) our previous studies with monocytes [26]; (2) the effects of LR-90 on renal nitrotyrosine in streptozotocin-diabetic rats [24]; and (3) our observation that the compound also attenuated the increased kidney expression of $C y b b$, the gene encoding for the gp91 ${ }^{\text {phox }}$ subunit of NADPH (data not shown), to assume that the LR-90 could also prevent renal oxidative stress in this animal model.

In addition to hyperglycaemia, untreated $\mathrm{ZDF}$ rats exhibited severe hyperlipidaemia and increased lipid peroxidation. Interestingly, we found the latter, measured as malondialdehyde in tissues, correlated well with urinary albumin excretion $(r=0.81, p<0.0001)$. Malondialdehyde has been widely recognised as an important marker of lipid peroxidation and has been shown to correlate strongly with the degree of renal damage in experimental and human diabetic nephropathy [47]. Experimental evidence linking hyperlipidaemia to renal injury and progression of renal fibrogenesis has been well documented; lipids can modulate the progression of chronic renal diseases and may even be primary factors in the pathogenesis of renal tissue injury [48, 49]. Additionally, the synergistic effects of hyperlipidaemia and diabetes on the development of renal injury have been recently observed in several animal models. In Apoe knockout mice, hyperlipidaemic animals with diabetes displayed a more extensive and severe degree of renal glomerular and tubulointerstitial injury as well as higher levels of urinary albumin excretion when compared with normolipidaemic diabetic animals [50]. Similarly, in obesityprone and spontaneously hypertensive hybrid rat models $(S H H F / G m i-f a \times L A / N-f a)$, the development of glomerulosclerosis and interstitial fibrosis was dependent on lipoxidation, as without hypercholesterolaemia, glycoxidation per se was not nephrotoxic [51]. Nonetheless, it remains unclear whether hyperglycaemia-driven AGE formation or lipids have a greater impact on the development of diabetic nephropathy in this animal model. Earlier studies in ZDF rats demonstrated that both elevated triacylglycerol and cholesterol, and progressive accumulation of lipids and their peroxidation products in the glomeruli and tubulointerstitium predate the development of glomerular and tubular damage in ZDF rats [14, 15], suggesting that hyperlipidaemia, in concert with hyperglycaemia, may be responsible for the 
increased oxidative stress and initiation of injury in the kidneys of these animals. Thus, it is logical to assume that the ability of LR-90 to lower plasma lipids, as well as preventing the accumulation of lipid peroxidation products in the kidney as demonstrated in the present studies, may have contributed to some extent to its renoprotective effects, independently of its inhibitory effects on AGE formation and AGER activation.

In summary, the present studies demonstrate that LR-90 retards the development of nephropathy in ZDF rats, concomitantly with marked inhibition of AGE formation and subsequent AGER production, a reduction in plasma lipids and kidney peroxides, and downregulation of expression of prosclerotic growth factors, matrix proteins and downstream intracellular signals such as p38 and p44/ 42 MAPKs, which are involved in glomerulosclerosis and renal fibrosis. Additionally, LR-90 downregulated activation of the inflammatory transcription factor NF- $\mathrm{kB}$. While the mechanisms by which LR-90 exerts its protective effects on the diabetic kidney are still not fully resolved, results to date are consistent with the proposed pleiotrophic effects of LR-90 as an AGE inhibitor, antioxidant, antifibrotic, hypolipidaemic and anti-inflammatory agent.

Acknowledgements The authors would like to thank M. Lee and B. Armstrong of the City of Hope Microscope Core Facility for their technical assistance and P. Chu of the Department of Anatomic Pathology for his helpful suggestions.

Duality of interest The authors declare that there is no duality of interest associated with this manuscript.

\section{References}

1. Friedman EA, Friedman AL (2007) Is there really good news about pandemic diabetic nephropathy? Nephrol Dial Transplant 22:681-683

2. Rossing P (2006) Diabetic nephropathy: worldwide epidemic and effects of current treatment on natural history. Curr Diab Rep 6:479-483

3. Lehmann R, Schleicher ED, Lehmann R, Schleicher ED (2000) Molecular mechanism of diabetic nephropathy. Clin Chim Acta 297:135-144

4. Raptis AE, Viberti G (2001) Pathogenesis of diabetic nephropathy. Exp Clin Endocrinol Diabetes 109(Suppl 2):S424-S437

5. Thomas MC, Forbes JM, Cooper ME (2005) Advanced glycation end products and diabetic nephropathy. Am J Ther 12:562-572

6. Bohlender JM, Franke S, Stein G, Wolf G (2005) Advanced glycation end products and the kidney. Am J Physiol 289:F645-F659

7. Horie K, Miyata T, Maeda K et al (1997) Immunohistochemical colocalization of glycoxidation products and lipid peroxidation products in diabetic renal glomerular lesions. J Clin Invest 100:2995-3004

8. Tanji N, Markowitz GS, Fu C et al (2000) Expression of advanced glycation end products and their cellular receptor RAGE in diabetic nephropathy and nondiabetic renal disease. J Am Soc Nephrol 11:1656-1666
9. Jensen LJ, Ostergaard J, Flyvbjerg A (2005) AGE-RAGE and AGE cross-link interaction: important players in the pathogenesis of diabetic kidney disease. Horm Metab Res 37(Suppl 1):26-34

10. Tan AL, Forbes JM, Cooper ME (2007) AGE, RAGE, and ROS in diabetic nephropathy. Semin Nephrol 27:130-143

11. Peterson RG Shaw WN, Neel MA et al (1990) Zucker diabetic fatty as a model for non-insulin dependent diabetes mellitus. ILAR News 32:16-19

12. Kasiske BL, O'Donnell MP, Keane WF (1992) The Zucker rat model of obesity, insulin resistance, hyperlipidemia, and renal injury. Hypertension 19(Suppl 1):I110-I115

13. Vora JP, Zimsen SM, Houghton DC, Anderson S (1996) Evolution of metabolic and renal changes in the ZDF/Drt-fa rat model of type II diabetes. J Am Soc Nephrol 7:113-117

14. Coimbra TM, Janssen U, Grone HJ et al (2000) Early events leading to renal injury in obese Zucker (fatty) rats with type II diabetes. Kidney Int 571:167-182

15. Chander PN, Gealekman O, Brodsky SV et al (2004) Nephropathy in Zucker diabetic fat rat is associated with oxidative and nitrosative stress: prevention by chronic therapy with a peroxynitrite scavenger ebselen. J Am Soc Nephrol 15:2391-2403

16. McCarthy KJ, Routh RE, Shaw W, Walsh K, Welbourne TC, Johnson JH (2000) Troglitazone halts diabetic glomerulosclerosis by blockade of mesangial expansion. Kidney Int 58:2341-2350

17. Baylis C, Atzpodien EA, Freshour G, Engels K (2003) Peroxisome proliferator-activated receptor $\gamma$ agonist provides superior renal protection versus angiotensin-converting enzyme inhibition in a rat model of type 2 diabetes with obesity. J Pharmacol Exp Ther 307:854-860

18. Mizuno M, Sada T, Kato M, Koike H (2002) Renoprotective effects of blockade of angiotensin II AT1 receptors in an animal model of type 2 diabetes. Hypertens Res 25:271-278

19. Wihler C, Schäfer S, Schmid K et al (2005) Renal accumulation and clearance of advanced glycation end-products in type 2 diabetic nephropathy: effect of angiotensin-converting enzyme and vasopeptidase inhibition. Diabetologia 48:1645-1653

20. Schäfer S, Linz W, Vollert H et al (2004) The vasopeptidase inhibitor AVE7688 ameliorates type 2 diabetic nephropathy. Diabetologia 47:98-103

21. Schrijvers BF, Flyvbjerg A, Tilton RG et al (2006) A neutralizing VEGF antibody prevents glomerular hypertrophy in a model of obese type 2 diabetes, the Zucker diabetic fatty rat. Nephrol Dial Transplant 21:324-329

22. Rahbar S, Figarola JL (2003) Novel inhibitors of advanced glycation endproducts. Arch Biochem Biophys 419:63-79

23. Rahbar S (2007) Novel inhibitors of glycation and AGE formation. Cell Biochem Biophys 48:147-157

24. Figarola JL, Scott S, Loera S et al (2003) LR-90, a novel advanced glycation endproduct inhibitor, prevents progression of diabetic nephropathy in streptozotocin-diabetic rats. Diabetologia 46:1140-1152

25. Figarola JL, Rahbar S, Scott S et al (2005) Prevention of early renal disease, dyslipidemia and lipid peroxidation in STZ-diabetic rats by LR-9 and LR-74, novel AGE inhibitors. Diabetes Metab Res Rev 21:533-544

26. Figarola JL, Shanmugam N, Natarajan R, Rahbar S (2007) Antiinflammatory effects of the advanced glycation end product inhibitor LR-90 in human monocytes. Diabetes 56:647-655

27. Wilkinson-Berka JL, Kelly DJ, Koerner SM et al (2002) ALT-946 and aminoguanidine, inhibitors of advanced glycation, improve severe nephropathy in the diabetic transgenic (mREN-2) rat. Diabetes 51:3283-3289

28. Ohga S, Shikata K, Yozai K et al (2006) Thiazolidinedione ameliorates renal injury in experimental diabetic rats through antiinflammatory effects mediated by inhibition of NF- $\mathrm{KB}$ activation. Am J Physiol 292:F1141-F1150 
29. Reckelhoff JF, Kanji V, Racusen LC et al (1998) Vitamin E ameliorates enhanced renal lipid peroxidation and accumulation of F2-isoprostanes in aging kidneys. Am J Physiol 274:R767-R774

30. Chuang L, Guh J (2001) Extracellular signals and intracellular pathways in diabetic nephropathy. Nephrology 6:165-172

31. Thomas MC, Atkins RC (2006) Blood pressure lowering for the prevention and treatment of diabetic kidney disease. Drugs 66:2213-2234

32. Song JH, Cha SH, Hong SB, Kim DH (2006) Dual blockade of the rennin-angiotensin system with angiotensin-converting enzyme inhibitors and angiotensin II receptor blockers in chronic kidney disease. J Hypertens Suppl 24:S101-S106

33. Davis BJ, Forbes JM, Thomas MC et al (2004) Superior renoprotective effects of combination therapy with $\mathrm{ACE}$ and AGE inhibition in the diabetic spontaneously hypertensive rat. Diabetologia 47:89-97

34. Wu YG, Lin H, Qian H et al (2006) Renoprotective effects of combination of angiotensin converting enzyme inhibitor with mycophenolate mofetil in diabetic rats. Inflamm Res 55:192-199

35. Forbes JM, Fukami K, Cooper ME (2007) Diabetic nephropathy: where hemodynamics meets metabolism. Exp Clin Endocrinol Diabetes 115:69-84

36. Mason RM, Wahab NA (2003) Extracellular matrix metabolism in diabetic nephropathy. J Am Soc Nephrol 14:1358-1373

37. Reeves WB, Andreoli TE (2000) Transforming growth factor beta contributes to progressive diabetic nephropathy. Proc Natl Acad Sci U S A 97:7667-7669

38. Wahab NA, Yevdokimova N, Weston BS et al (2001) Role of connective tissue growth factor in the pathogenesis of diabetic nephropathy. Biochem J 359:77-87

39. Pugliese G, Pricci F, Romeo G et al (1997) Upregulation of mesangial growth factor and extracellular matrix synthesis by advanced glycation end products via a receptor-mediated mechanism. Diabetes 46:1881-1887

40. Zhou G, Li C, Cai L (2004) Advanced glycation end-products induce connective tissue growth factor-mediated renal fibrosis predominantly through transforming growth factor $\beta$-independent pathway. Am J Pathol 165:2033-2043

41. Crean JK, Finlay D, Murphy M et al (2002) The role of p42/44 MAPK and protein kinase B in connective tissue growth factor induced extracellular matrix protein production, cell migration, and actin cytoskeletal rearrangement in human mesangial cells. J Biol Chem Nov 277:44187-44194

42. Fujita H, Omori S, Ishikura $\mathrm{K}$ et al (2004) ERK and p38 mediate high-glucose-induced hypertrophy and TGF- $\beta$ expression in renal tubular cells. Am J Physiol 286:F120-F126

43. Zill H, Bek S, Hofmann T et al (2003) RAGE-mediated MAPK activation by food-derived AGE and non-AGE products. Biochem Biophys Res Commun 300:311-315

44. Yeh CH, Sturgis L, Haidacher J et al (2001) Requirement for p38 and p44/p42 mitogen-activated protein kinases in RAGE-mediated nuclear factor-kappaB transcriptional activation and cytokine secretion. Diabetes 50:1495-1504

45. Lee HB, Yu MR, Yang Y, Jiang Z, Ha H (2003) Reactive oxygen species-regulated signaling pathways in diabetic nephropathy. J Am Soc Nephrol 14(Suppl 3):S241-S245

46. Suzaki Y, Ozawa Y, Kobori H (2006) Intrarenal oxidative stress and augmented angiotensinogen are precedent to renal injury in Zucker diabetic fatty rats. Int J Biol Sci 3:40-46

47. Chang JM, Kuo MC, Kuo HT et al (2005) Increased glomerular and extracellular malondialdehyde levels in patients and rats with diabetic nephropathy. J Lab Clin Med 146:210-215

48. Abrass CK (2004) Cellular lipid metabolism and the role of lipids in progressive renal disease. Am J Nephrol 24:46-53

49. Rosario RF, Prabhakar S (2006) Lipids and diabetic nephropathy. Curr Diab Rep 6:455-462

50. Lassila M, Seah KK, Allen TJ et al (2004) Accelerated nephropathy in diabetic apolipoprotein e-knockout mouse: role of advanced glycation end products. J Am Soc Nephrol 15:2125-2138

51. Dominguez JH, Tang N, Xu W et al (2000) Studies of renal injury III: lipid-induced nephropathy in type II diabetes. Kidney Int 57:92-104 